\title{
Una aplicación del análisis espacial al estudio de las diferencias regionales del ingreso en México
}

\author{
Carlos J. Vilalta y Perdomo*
}

\begin{abstract}
Based on three different statistical techniques, we confirm in this work the existence of an economical convergence trend between 1970 and 1980 and of a divergence one between 1970 and 1990. Our principal conclusions are as follows: (1) the regional divergence in the country has been present during this twenty year period. However, an spatial analysis of the process shows that the regional inequalities have decreased, and at the same time the growth has been spatially concentrated. This suggests the existence of convergence clubs and the methodological convenience of an spatial analysis if the development. (2) The periods between 1895-1960 and 1970-1990 are particularly different in regard to the explicative variables of regional differences. (3) After the 1980 's crisis, the states with a better economy are either geographically located in the border with the United States of America or those where tourism or oil extraction are important for their economy.
\end{abstract}

Keywords: Mexico, economical development, regional divergence.

\section{Resumen}

Con base en tres técnicas estadísticas diferentes, este trabajo confirma una tendencia de convergencia económica entre 1970 y 1980 y de divergencia económica entre 1970 y 1990. Las conclusiones principales son: (1) que la divergencia regional dentro del país ha estado presente durante este periodo de veinte años, pero que analizado el proceso con el uso de técnicas de análisis espacial, se observa que las desigualdades regionales han tendido a disminuir y que simultáneamente el crecimiento ha estado espacialmente concentrado, sugiriendo los dos puntos anteriores: la existencia de clubes de convergencia y la conveniencia metodológica de analizar espacialmente el desarrollo; (2) que los periodos 1895-1960 y 1970-1990 son marcadamente diferentes en cuanto a las variables explicativas de las diferencias regionales, y (3) que después de la crisis de los ochenta los estados con una mejor condición económica son precisamente aquellos que geográficamente se hallan en la frontera con los Estados Unidos o aquellos en donde el turismo o la extracción de petróleo han sido fundamentales para su economía.

Palabras clave: México, desarrollo económico, divergencia regional

* Instituto Tecnológico y de Estudios Superiores de Monterrey, campus Ciudad de México. Correo-e: carlos.vilalta@itesm.mx 


\section{Introducción}

El debate sobre las razones de la convergencia o divergencia económica regional se ha ilustrado principalmente con base en las experiencias de países desarrollados. Sin embargo, estas experiencias no necesariamente coinciden con: (1) las tendencias observadas en todos los países occidentales, (2) ni con la realidad de los países en desarrollo. Por ejemplo, para el caso mexicano, Lemoine (1973) encontró que en el periodo 1895-1960 las desigualdades regionales en el ingreso per cápita (IPC) aumentaron, mientras que Sala-i-Martín (1996), entre muchos otros, encontraría lo opuesto para el caso de los Estados Unidos.

El estudio de las tendencias económicas regionales son de interés académico y de relevancia social. Por un lado, hay un interés teórico en entender cómo las regiones se desenvuelven económicamente, especialmente en un esquema actual de competencia global. Por otro lado, los beneficios sociales procedentes del análisis de la evidencia empírica proveniente de una economía en desarrollo, como en el caso de México, son claros. Esta información se puede utilizar para el diseño y aplicación de políticas desarrollo económico regionales y nacionales. Por lo anterior, el objetivo de este artículo es comparar estudios previos y ofrecer más evidencia dentro del debate convergencia-divergencia con base en la experiencia de México.

Este trabajo se basa metodológicamente en tres técnicas similares que miden aspectos diferentes: (a) estadísticas descriptivas y un análisis de regresión de mínimos cuadrados (Messmacher, 2000), (b) un análisis de autocorrelación espacial, y (c) un análisis de correlación para variables ordinales (Lemoine, 1973). Las dos primeras tienen por objeto probar las diferentes hipótesis de convergencia (sigma y beta) durante el periodo 1970-1990. La última se utiliza para medir el nivel de asociación entre el IPC y otras variables teóricamente determinantes del desarrollo económico regional; en este último caso nos basamos en el estudio previo de Lemoine para llegar a resultados consistentes y comparables para el periodo 1895-1990. El documento está dividido de la siguiente manera: Estudios previos, metodología e información estadística, resultados y discusión, y conclusiones. 


\section{Estudios previos}

Sala-i-Martín (1996) ha argumentado que algunos países occidentales han logrado atenuar las diferencias en los niveles de IPC entre sus regiones. Para el caso de los Estados Unidos, este autor ha encontrado evidencia a largo plazo de convergencia en el ingreso personal entre estados en el periodo 1880-1990. Esta tendencia ha sido documentada extensivamente (Carlino y Mills, 1996; Hoover y Giarratani, 1984; Coelho y Ghali, 1971). Sala-iMartín también hace una distinción entre dos tipos de convergencia regional: s-convergence (convergencia sigma), la cual sugiere que las diferencias de IPC entre regiones tienden a decaer a través del tiempo, y b-convergence (convergencia beta), la cual sugiere que las economías pobres tienden a crecer más rápido que las economías ricas. Este autor encuentra que ambos tipos de convergencia están presentes en una reducida pero importante muestra de países en la Unión Europea (Alemania, Reino Unido, Italia y España), los Estados Unidos, Canadá y Japón. También encuentra que la velocidad en la convergencia (tasa de $2 \%$ anual) es muy similar entre estos países. Aunque debe mencionarse que las diferencias en el ingreso es mayor entre las regiones europeas que entre los estados de Estados Unidos (Puga, 1999).

En Suecia, Persson (1997) también muestra evidencia estadística de una tendencia a la convergencia regional en los niveles de IPC. Encuentra evidencia a largo plazo observando el desarrollo económico en los condados suecos en el periodo 1911 a 1993 . Y, coincidentemente, la tasa anual de convergencia regional es igual a la de Sala-i-Martín.

Sin embargo, de acuerdo con Quah (1996a), estos resultados deben tomarse con precaución. Según este autor, “el IPC en las regiones europeas no es el mismo ni se ha mantenido constante; la distribución en el ingreso regional ha fluctuado a través del tiempo" (Quah, 1996a: 951). El punto principal de su argumento es que las regiones no deben ser estudiadas independientemente, como es el caso de la mayoría de los análisis de regresión o modelos de series de tiempo. Más bien, estos análisis deben tener una perspectiva estructural dinámica. La localización geográfica y los efectos de regiones vecinas (clusters) son fundamentales para explicar el desarrollo de una región (Quah, 1996b). Desde esta perspectiva, el autor argumenta que Europa en realidad ofrece evidencia de un proceso de divergencia económica regional. En particular, argumenta la existencia de convergence clubs, los cua- 
les son grupos de regiones que están despegándose del resto de Europa en materia de IPC; esto con la excepción de los estados de la Unión Americana, los cuales sí exhiben evidencia de convergencia en el IPC "sin ambigüedad hasta donde lo permite el error muestral” (Quah, 1996b: 1353).

Esta perspectiva de dependencia interregional parece ser válida con base en la afirmación de Kollman (1995: 437) de que "las correlaciones entre regiones en el crecimiento de la producción de una industria específica son usualmente más fuertes que las correlaciones entre industrias diferentes en el crecimiento de la productividad dentro de una misma región". Para efectos metodológicos, esto significa que el crecimiento y la expansión geográfica de un sector industrial no es espacialmente aleatorio, sino que existe una geografía industrial en donde las regiones no son observaciones independientes estadísticamente hablando. Lo anterior tiene implicaciones metodológicas importantes dentro del análisis estadístico (Anselin, 1988). Se debe entender que existe una dinámica económica industrial o sectorial que supera las fronteras político-administrativas. Debe comentarse que Kollman encuentra que el crecimiento en la productividad esta más fuertemente correlacionado entre estados de la unión americana que entre los países del grupo de los 7 (G-7).

Magrini (1999) muestra evidencia en favor de la hipótesis de divergencia regional. Este autor registra una tendencia hacia la desigualdad en el periodo 1979-1990 en un estudio de 122 regiones funcionales en Europa. Aunque su periodo de estudio es menor al de los artículos anteriores, el autor argumenta que es tiempo suficiente para considerar movimientos cíclicos en el comportamiento económico. La diferencia en sus hallazgos se basa fundamentalmente en su definición de región; este autor utiliza regiones funcionales europeas (la región como una esfera de influencia socio-económica), las cuales han sido predefinidas en la investigación geográfica previa de Hall y Hay (1980) sobre centros de desarrollo europeos.

En el caso de Japón, Fujita y Tabuchi (1997) encuentran una tendencia hacia el "incremento en las diferencias del ingreso entre regiones centrales y periféricas”. Los autores argumentan que hasta cierto punto esto se puede explicar con base en la tendencia actual a la concentración de conocimiento intensivo (knowledgeintensive) en las regiones centrales y en la dispersión de la producción japonesa en masa (mass-production) a las regiones periféricas del país o al mundo en desarrollo. Precisamente relacionado 
con lo anterior, Leach (1996: 429) explica que "las desigualdades en el ingreso promedio entre regiones se deben a diferencias en los sueldos los cuales son reforzados por las diferencias regionales en la estructura del empleo".

También en relación con lo anterior, Paci y Pagliaru (1997) argumentan que un proceso de convergencia regional depende de un cambio económico estructural, el cual se define como: (1) un proceso continuo, (2) no mecánico, y (3) posiblemente limitado al cambio transitorio de una economía agrícola a una de manufacturas. Estos autores argumentan que en para el caso italiano no hay evidencia para demostrar una convergencia teórica del tipo beta (b-convergence), en la cual las regiones pobres tienden a crecer más rápidamente que las regiones más prósperas.

En el caso mexicano, se han identificado procesos discordantes según los periodos de análisis. En específico, se han registrado procesos de: (1) divergencia durante el periodo 1895-1960 (Lemoine, 1973); (2) divergencia durante 1900-1970 para revertirse en esa última década 1970-1980 (Hernández, 1984); (3) convergencia entre 1980-1985, pero en sumatoria de divergencia para todo el periodo 1980-1999 (Arroyo, 2001), y (4) convergencia entre 1970-1985 y 1993-1999, de divergencia entre 1985-1993, pero en sumatoria de divergencia para el periodo de 1970-1999 (Messmacher, 2000). En síntesis, parece ser que durante la primera mitad del siglo xx, México siguió un proceso acorde con la teoría de Myrdal (1957), la cual señala que en etapas de acumulación capitalista industrial se presenta inicialmente un fenómeno de crecimiento regional de la riqueza desbalanceado. Pero para el periodo 1970 en adelante, la literatura muestra un proceso inarmónico de altas y bajas, convergencia y divergencia, no predichos por la teoría.

En esta lógica de probar teorías desde una perspectiva histórica a largo plazo, Lemoine calculó coeficientes de correlación entre el IPC de 1960 (variable dependiente, VD) con otras variables determinantes del desarrollo industrial (variables independientes, vI) para cada una de las respectivas décadas (1895-1960). Su hipótesis y conclusión principal es que los cambios pasados en el comportamiento de esas vi pueden explicar los niveles de IPC, al menos hasta 1960. Al probar esta hipótesis, Lemoine encontró que esta tendencia a la divergencia regional estaba históricamente correlacionada precisamente con diferenciales en los procesos de industrialización, urbanización, niveles de inversión pública, etc. entre estados. Esto se concluye también en el estudio de largo 
plazo entre 1900-1980 de Hernández (1984). Por lo que no parece haber debate a este respecto. Pero desde 1970 la desigualdad regional en el IPC se ha vuelto difícil de predecir y es materia de análisis detallado.

Inicialmente, en el cuadro 1 se puede observar una tendencia a la disparidad regional con base en los incrementos en el coeficiente de variación del IPC entre 1970 y 1990. El cuadro 1 muestra que la disparidad en el ingreso entre las diferentes entidades federativas aumentó principalmente durante la década de los ochenta. También hay más entidades por debajo del IPC promedio que por arriba. Durante los ochenta, sólo hubieron 2 entidades que no cambiaron en su ranking: 13 subieron y 17 bajaron. En suma, 15 entidades aumentaron su IPC, mientras que las 17 restantes experimentaron una disminución. Pero entre 1970 y 1990, sólo una entidad vio su IPC disminuir ligeramente (Veracruz).

El cuadro 1 muestra de manera a priori que México ha experimentado un proceso de divergencia en el IPC entre sus entidades federativas durante 1970-1990. El coeficiente de variación nos muestra un sostenimiento en las desigualdades entre 1970 y 1980 , y de divergencia entre 1980-1990. No hay diferencias visibles en relación con los estudios previos. Sin embargo, algo claro es que el cálculo derivado de la media y la desviación estándar no es prueba suficiente ni contribuye determinantemente en el estudio de las tendencias económicas regionales; la observación de la variación es sólo parte inicial. Para contribuir de manera efectiva a la literatura especializada en México y al debate en general, en este trabajo realizamos un análisis estadístico más elaborado para el caso mexicano, utilizando varias perspectivas metodológicas con el fin de reunir evidencia y aportar significativamente al debate sobre convergencia y divergencia regional.

\section{Metodología e información estadística}

Ya que este trabajo persigue comparar resultados de diferentes trabajos y agregar evidencia, se utilizan tres técnicas diferentes: (1) un análisis de regresión de mínimos cuadrados, (2) un análisis de autocorrelación espacial, y (3) un análisis de correlación de Spearman.

Por un lado, las primeras dos técnicas se enfocan a probar las hipótesis sigma y beta de convergencia regional. La técnica de correlación de Spearman se utiliza para analizar las variables aso- 


\section{Cuadro 1}

Ingreso per cápita (IPC) en México*

\begin{tabular}{|l|c|c|c|}
\hline \multicolumn{1}{|c|}{ Entidad Federativa } & $\mathbf{1 9 7 0}$ & $\mathbf{1 9 8 0}$ & $\mathbf{1 9 9 0}$ \\
\hline Aguascalientes & 562 & 795 & 747 \\
\hline Baja California & 1,040 & 1,286 & 1,268 \\
\hline Baja California Sur & 928 & 1,280 & 1,425 \\
\hline Campeche & 589 & 818 & 4,111 \\
\hline Chiapas & 328 & 594 & 372 \\
\hline Chihuahua & 672 & 961 & 1,099 \\
\hline Coahuila & 832 & 1,193 & 1,374 \\
\hline Colima & 606 & 795 & 1,307 \\
\hline Distrito Federal & 1,364 & 2,260 & 2,604 \\
\hline Durango & 488 & 757 & 700 \\
\hline Estado de México & 747 & 1,001 & 751 \\
\hline Guanajuato & 535 & 664 & 714 \\
\hline Guerrero & 374 & 555 & 607 \\
\hline Hidalgo & 365 & 667 & 557 \\
\hline Jalisco & 725 & 1,039 & 1,030 \\
\hline Michoacán & 365 & 576 & 478 \\
\hline Morelos & 578 & 799 & 1,090 \\
\hline Nayarit & 520 & 758 & 706 \\
\hline Nuevo León & 1,157 & 1,616 & 1,775 \\
\hline Oaxaca & 245 & 407 & 372 \\
\hline Puebla & 436 & 658 & 520 \\
\hline Querétaro & 549 & 838 & 844 \\
\hline Quintana Roo & 685 & 1,218 & 1,359 \\
\hline San Luis Potosí & 423 & 576 & 589 \\
\hline Sinaloa & 651 & 781 & 871 \\
\hline Sonora & 951 & 1,091 & 1,574 \\
\hline Tabasco & 495 & 1,295 & 966 \\
\hline Tamaulipas & 307 & 1,001 & 1,140 \\
\hline Tlaxcala & 649 & 618 & 430 \\
\hline Veracruz & 506 & 712 & 647 \\
\hline Yucatán & 484 & 707 \\
\hline Zacatecas & 0.37 & 700 \\
\hline Promedio & & 1,025 \\
\hline Coeficiente de & & 0.46 \\
\hline Desigualdad** & & \\
\hline & & & \\
\hline & & & \\
\hline
\end{tabular}

Fuente: Garza y Rivera (1994)

* En dólares americanos de 1970

** Producto de dividir la desviación estándar entre el promedio. El coeficiente de 1990 no incluye al estado de Campeche, el cual muestra un elevado crecimiento en su ingreso per cápita debido al boom petrolero. Incluyendo Campeche, el coeficiente se eleva a .70 (Garza y Rivera, 1994) 
ciadas a la desigualdad regional y complementar históricamente el estudio histórico previo de Lemoine. Por otro lado, las técnicas de regresión y de correlación de Spearman ya han sido utilizadas en la literatura especializada mexicana. La técnica de autocorrelación espacial no ha sido encontrada en la revisión de la literatura nacional e internacional. Sin embargo esta técnica ya ha sido aplicada en México dentro del estudio de la geografía electoral (Vilalta, 2003).

La definición de región en este estudio corresponde a la de entidad federativa. Esto se debe a la necesidad y conveniencia de usar la misma unidad de análisis que previos autores han utilizado en sus estudios. Este análisis histórico es inicialmente válido, ya que no han habido cambios en las geografías estatales. Sin embargo, hay dos limitaciones muy importantes que deben mencionarse. Una fundamental es que entidad federativa o región política no es sinónimo de región económica, cuestión que limita la capacidad explicativa del análisis. ${ }^{1}$ Otra limitación importante es que no fue posible conseguir información económica sobre ciertas variables para cada una de las décadas, por lo que algunos coeficientes no fueron calculados.

\section{Cuadro 2 \\ Variables en el análisis*}

\begin{tabular}{|c|c|c|c|}
\hline $\begin{array}{c}\text { Variables } \\
\text { dependientes }\end{array}$ & Operacionalización & $\begin{array}{c}\text { Variables } \\
\text { independientes }\end{array}$ & Operacionalización \\
\hline $\begin{array}{l}\text { Ingreso per } \\
\text { cápita }\end{array}$ & $\begin{array}{l}\text { Ingreso per cápita } \\
(1970,1980,1990)\end{array}$ & Urbanización & $\begin{array}{l}\text { Porcentaje de población } \\
\text { en localidades de } 15 \text { mil o } \\
\text { más habitantes ( } 1970 \text {, }\end{array}$ \\
\hline $\begin{array}{l}\text { Cambio en el } \\
\text { ingreso per } \\
\text { cápita }\end{array}$ & $\begin{array}{l}\text { Tasa de cambio en el } \\
\text { IPC entre } 1970 \text { y } 1990\end{array}$ & $\begin{array}{l}\text { Sector transporte } \\
\text { Sector agricultura } \\
\text { Alfabetización }\end{array}$ & $\begin{array}{l}\text { Empleados en el sector } \\
\text { transporte como } \\
\text { porcentaje de la fuerza de } \\
\text { trabajo total (1980, 1990) } \\
\text { Empleados en el sector } \\
\text { agrícola como porcentaje } \\
\text { de la fuerza de trabajo } \\
\text { total (1980, 1990) } \\
\text { Población de } 15 \text { años o } \\
\text { más que pueden leer y } \\
\text { escribir }(1980,1990)\end{array}$ \\
\hline
\end{tabular}

"La variable dependiente fue transformada en varias ocasiones a una escala logarítmica; en otra ocasión, fue transformada en variable ordinal. Elaboración propia.

\footnotetext{
${ }^{1}$ Una perspectiva iluminativa sería el análisis de las diferencias en el IPC a partir de una redefinición de región funcional como es el caso de Magrini (1999) y Hall y Hay (1980), y una perspectiva diferente sería el análisis de las diferencias en el IPC en el sistema de ciudades.
} 
Estas variables tienen una relación con el desarrollo económico por dos razones fundamentales. Primero, todas ellas están empírica y teóricamente correlacionadas con los niveles de ingreso. Segundo, los estados mexicanos con mejor desempeño económico, en términos de crecimiento, han mostrado cambios históricos favorables en cada una de éstas, mientras que lo opuesto ha sucedido en las regiones más pobres (Lemoine, 1973). En otras palabras, mientras que un estado posea una mayor cantidad de cada una de estas variables -excepto por fuerza laboral en actividades agrícolas-, mayor será su IPC. Estas variables han explicado estadísticamente de manera favorable niveles pasados de IPC.

La fuente de información en cuanto a transporte, agricultura y alfabetización corresponde a los censos de población de 1980 y 1990. Esta información está disponible en el sistema SIMBAD en el sitio web del Instituto Nacional de Estadística, Geografía e Informática (INEGI). La información respecto a IPC y niveles de urbanización fueron obtenidos del estudio de Garza y Rivera (1994) sobre la dinámica macroeconómica de las ciudades en México.

\section{Resultados}

\subsection{Sobre las hipótesis de convergencia regional}

Para probar los dos tipos de convergencia regional de Sala-i-Martín (1996), utilizamos la comparación de las desviaciones estándar en tiempos diferentes (convergencia $\sigma$ ) y un análisis de regresión de convergencia (convergencia $\beta$ ) similar al de Messmacher (2000). ${ }^{2}$ El cuadro 3 indica en detalle qué tipo de técnica corresponde a cada prueba a realizarse.

\section{Cuadro 3}

Técnicas para las pruebas sobre los diferentes tipos de convergencia

\begin{tabular}{|l|l|c|l|}
\hline \multicolumn{1}{|c|}{ Prueba } & \multicolumn{1}{|c|}{ Técnica } & Resultado & \multicolumn{1}{c|}{ Conclusión } \\
\hline $\begin{array}{l}\text { Convergencia } \\
\text { sigma }\end{array}$ & $\begin{array}{l}\text { Cálculo de la desviación } \\
\text { estándar del IPC }(1970 \mathrm{a} \\
1990)\end{array}$ & $\sigma_{1}>\sigma_{2}>\sigma_{3}$ & Convergencia regional \\
\hline $\begin{array}{l}\text { Convergencia } \\
\text { beta }\end{array}$ & $\begin{array}{l}\text { Regresión de } \\
\text { convergencia } \beta\end{array}$ & $\begin{array}{l}\beta(+) \\
\beta(-)\end{array}$ & $\begin{array}{l}\text { Divergencia regional } \\
\text { Convergencia regional }\end{array}$ \\
\hline
\end{tabular}

Fuente: Elaboración propia.

${ }^{2}$ En esta parte del análisis se incluyó o excluyó al estado de Campeche para hacer los resultados comparables con estudios previos y para comparar la importancia de este estado. 
En primer lugar, el cálculo de la desviación estándar para el periodo 1970 a 1990 nos muestra que las diferencias en el ingreso en el conjunto de las entidades federativas se redujo en 1980 frente a 1970, para luego aumentar en 1990 (véanse los cuadros 4 y 5). Estos resultados demuestran una tendencia de 30 años a la divergencia económica regional. Es decir, la evidencia apunta a rechazar la hipótesis de convergencia sigma, la cual sugiere que las diferencias de IPC entre regiones tienden a decaer a través del tiempo; con base en nuestros datos, ha ocurrido lo contrario.

\section{Cuadro 4}

Prueba de convergencia sigma: desviación estándar y sesgo del IPC para el periodo 1970 a 1990*

\begin{tabular}{|l|c|c|c|}
\hline & $\mathbf{1 9 7 0}$ & $\mathbf{1 9 8 0}$ & $\mathbf{1 9 9 0}$ \\
\hline Desviación estándar & 266 & 380 & 481 \\
\hline
\end{tabular}

* El cálculo se realiza sobre los logaritmos naturales (ln). $\mathrm{N}=31$ excluyendo a Campeche. Elaboración propia

\section{Cuadro 5}

Prueba de convergencia sigma: desviación estándar y sesgo del IPC para el periodo 1970 a 1990*

\begin{tabular}{|c|c|c|c|}
\hline & $\mathbf{1 9 7 0}$ & $\mathbf{1 9 8 0}$ & $\mathbf{1 9 9 0}$ \\
\hline Desviación estándar & 258 & 368 & 721 \\
\hline
\end{tabular}

* El cálculo es sobre los logaritmos naturales $(\ln ) . \mathrm{N}=32$, en este caso incluyendo a Campeche. Elaboración propia.

En segundo lugar, el análisis de regresión para la prueba de la convergencia beta, es decir, en la cual los estados más pobres tiendes a crecer más rápido que los estados ricos, toma la siguiente forma (Messmacher, 2000):

$$
\left(\ln y_{t}-\ln y_{t-1}\right)=\beta_{0}+\beta_{1} \ln y_{t-1}+\varepsilon_{t}
$$

donde:

$\ln =$ logaritmo natural, $\mathrm{y}_{\mathrm{t}}=$ IPC en 1990, $\mathrm{y}_{\mathrm{t}-1}=$ IPC en 1970, $\beta_{0}$ $=$ constante, $\beta_{1}=$ coeficiente y $\varepsilon_{\mathrm{t}}=$ error aleatorio

El sentido de la prueba es que si los niveles de IPC en 1990 están asociados negativamente con los niveles de IPC en 1970, entonces las entidades más pobres son las que han tendido a crecer más rápidamente, lo cual estaría en concordancia con la hipótesis de 
convergencia beta. Si al contrario, el coeficiente de correlación muestra un sentido positivo, entonces lo que ha sucedido es que las entidades federativas más ricas son las que han crecido más rápidamente, sugiriendo entonces el rechazo de la hipótesis de convergencia regional.

\section{Cuadro 6}

Prueba de convergencia beta: resultados de la regresión*

\begin{tabular}{|l|c|c|c|c|}
\hline & b & Beta & t & Sigma \\
\hline Constante & .229 & & .415 & .681 \\
\hline IPC en $1970(\ln )$ & .026 & .054 & .294 & .771 \\
\hline
\end{tabular}

"Variable dependiente: diferencia en el IPC entre 1970 y 1990 (ln). N = 31, excluyendo a Campeche. Elaboración propia.

\section{Cuadro 7}

Prueba de convergencia beta: resultados de la regresión*

\begin{tabular}{|l|c|c|c|c|}
\hline & b & Beta & t & Sigma \\
\hline Constante & .227 & & .239 & .812 \\
\hline IPC en $1970(\ln )$ & .033 & .041 & .224 & .824 \\
\hline
\end{tabular}

*Variable dependiente: diferencia en el IPC entre 1970 y 1990 (ln). N = 32, en este caso incluyendo a Campeche. Elaboración propia.

Los resultados que se muestran en los cuadros 6 y 7 indican que no hay relación estadísticamente significativa entre el IPC en 1970 con el cambio en IPC entre 1970 y $1990(B=.054$, n.s. y $B=.041$, n.s.). Es decir, no hay evidencia de que niveles previos de IPC hayan abierto o cerrado las brechas de ingreso entre los estados mexicanos; no hay relación, o bien la relación es muy pequeña, para ser considerada como estadísticamente probable. Sin embargo, cabe aclarar que los resultados sobre la significancia estadística de este coeficiente son irrelevantes, ya que estamos tratando sobre el cálculo de correlaciones de todas las unidades de análisis en cuestión (véase el cuadro 7), no de una muestra, por lo que se incorpora, también, el hecho de que los supuestos clásicos del muestreo aleatorio no aplican (Quah, 1996b). Invariablemente, los coeficientes no estandarizados (b) y estandarizados (Beta) son muy pequeños en ambos casos.

En síntesis, la evidencia que encontramos para rechazar la hipótesis de convergencia sigma (véanse los cuadros 4 y 5), junto con la nimia evidencia para rechazar la hipótesis de convergencia 
beta (véanse los cuadros 6 y 7) nos sugiere aceptar la hipótesis de divergencia regional en México durante 1970 y 1990. Estos resultados no son contradictorios a los previamente encontrados por Messmacher (2000) y Arroyo (2001). ${ }^{3}$

\subsection{Análisis espacial de la convergencia regional}

Para proseguir sistemáticamente con las pruebas de hipótesis, en este apartado se calcularon coeficientes de autocorrelación espacial "I" de Morán (1950). En este caso, el objetivo específico es probar la hipótesis de que el IPC está: (1) concentrado espacialmente, y (2) medir los cambios temporales en los niveles de concentración. La lógica del procedimiento es que un aumento temporal en la magnitud de los coeficientes indicaría un proceso de divergencia regional, mientras que una disminución indicaría evidencia de convergencia regional (véase el cuadro 8).

\section{Cuadro 8}

Técnica de análisis espacial para la prueba sobre convergencia regional

\begin{tabular}{|c|l|c|c|}
\hline \multicolumn{1}{|c|}{ Prueba } & \multicolumn{1}{|c|}{ Técnica } & Resultado & Conclusión \\
\hline Convergencia & $\begin{array}{l}\text { Coeficientes de } \\
\text { autocorrelación espacial }\end{array}$ & $I_{1}>I_{2}>I_{3}$ & Convergencia regional \\
\hline
\end{tabular}

Fuente: Elaboración propia.

Los resultados del coeficiente de Morán pueden variar entre +1 y -1. En el primer caso, mostraría una autocorrelación positiva, indicando una concentración espacial perfecta del ingreso; mientras que en el segundo caso mostraría una autocorrelación negativa, indicando una perfecta dispersión del ingreso. ${ }^{4}$ Un valor de cero indicaría un arreglo espacial perfectamente aleatorio (Morán, 1950).

La fórmula de autocorrelación espacial de Morán (1950) toma la siguiente forma:

\footnotetext{
${ }^{3}$ En una regresión que se calculó para la diferencia en el IPC entre 1970 y 1980 como VD y el IPC en 1970 como VI, ambas como logaritmos naturales, igualmente se mostró la no significancia estadística, pero un coeficiente mayor y positivo $(B=.215$, n.s.) sugiriendo, entonces, un proceso de divergencia regional entre 1970 y 1980.

${ }^{4}$ Una dispersión espacial perfecta de una variable sería similar en forma a los cuadros de un tablero de ajedrez.
} 


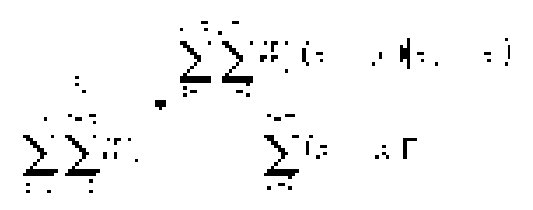

donde:

- $n$ es el número de elementos (estados) en la matriz de distancia-vecinos;

- $W_{i j}$ es la matriz de vecinos que define si un estado $i$ y $j$ son vecinos o no (el denominador da el número total de vecinos);

- $x$ es la variable en cuestión, en este caso ingreso per cápita.

En esta investigación se definieron a los estados vecinos de dos maneras diferentes: vecinos de primer orden y vecinos de primer orden ajustados. En el primer caso, la matriz de vecinos considera únicamente como tales a aquellas unidades geográficas contiguas. En el segundo caso, el procedimiento sigue dos pasos: primero la matriz incluye como vecinas a aquellas unidades que son contiguas; posteriormente, calcula el promedio de distancias (centroide a centroide) que hay en el conjunto de unidades geográficas, e incluye como vecinas a aquellas unidades que están por debajo de ese promedio. La razón de realizar este ajuste es que el criterio estricto de contigüidad no considera otra dimensión espacial fundamental, que es la cercanía física; dos unidades geográficas pueden estar muy cercanas físicamente, pero no ser contiguas.

El cuadro 9 muestra los coeficientes de autocorrelación espacial en el IPC durante el periodo 1970 a $1990 .{ }^{5}$ El coeficiente es sobre la tasa de cambio en el ingreso durante 1970-1990. En este caso siempre se incluyó como unidad geográfica a Campeche. La razón básica es que es metodológicamente incoherente dentro del análisis espacial borrar un estado del mapa. Las unidades geográficas, como dice Quah (1996b: 1360): "representan a gente real [...] no observaciones que pueden ser borradas para su utilidad en el análisis estadístico".

Para el primer caso de definición de vecindad de primer orden o estricta contigüidad, los resultados (véase el cuadro 9) sugieren que el IPC estaba moderadamente concentrado en $1970(I=.180$, $\mathrm{p}<.05)$, para luego descender en su concentración en 1990 ( $I=$ .130, n.s.). Entre 1980 y 1990 la concentración aumentó, sugiriendo en este caso una coincidencia con las tesis de periodos

${ }^{5}$ Se utilizó el software geográfico ArcView con la extensión de estadística espacial de S-Plus. 
cambiantes de convergencia-divergencia de Messmacher (2000) y Arroyo (2001). El cambio en el IPC de 1970 a 1990 ( $\Delta$ IPC 70-90) muestra casi un arreglo espacial aleatorio, sugiriendo que los cambios en los estados fueron independientes de los estados vecinos.

Cuadro 9

Resultados del análisis de autocorrelación espacial*

\begin{tabular}{|c|c|c|c|c|}
\hline \multicolumn{5}{|c|}{ Vecinos de primer orden } \\
\hline & $I P C 1970$ & $I P C 1980$ & $I P C 1990$ & $\Delta I P C 70-90$ \\
\hline Coeficiente $I$ & 0.180 & 0.101 & 0.130 & 0.024 \\
\hline Sig. & 0.048 & 0.214 & 0.130 & 0.601 \\
\hline \multicolumn{5}{|c|}{ Vecinos de primer orden ajustado } \\
\hline Coeficiente $I$ & 0.323 & 0.226 & 0.255 & 0.211 \\
\hline Sig. & 0.000 & 0.003 & 0.001 & 0.005 \\
\hline
\end{tabular}

"Incluye Campeche. Nota: Elaborado con los datos del cuadro 1. La significancia estadística es una prueba $\mathrm{Z}$ de dos colas sobre una distribución teóricamente normal.

Para el caso del análisis realizado bajo la definición de vecinos de primer orden ajustado, podemos ver que la concentración espacial en el IPC también disminuyó ligeramente entre 1970 ( $I=.323$, p <.01) y $1990(I=.255, \mathrm{p}<.01)$. Sin embargo, a diferencia del caso anterior, el cambio en el IPC entre 1970 y 1990 sí nos muestra una autocorrelación positiva o de concentración $(I=.239, \mathrm{p}$ $<.01$ ), indicando que aquellos estados que aumentaron más rápidamente su IPC eran aquellos que estaban rodeados o que eran vecinos de otros estados, también con aumentos relativos en su IPC; es decir, las tasas de crecimiento han sido espacialmente dependientes. ${ }^{6}$ Esta disminución moderada en la concentración espacial del IPC en el periodo de estudio, junto con una evidente concentración del cambio ( $\Delta$ IPC 70-90) nos sugiere que México es un país con regiones económicas claramente diferenciadas e independientes en sus dinámicas; el país muestra tener clusters de crecimiento o "clubes de convergencia" (Quah, 1996b: 1355). ${ }^{7}$

Como se aprecia, no hay desacuerdo en el hecho de que el IPC ha estado concentrado espacialmente. Pero dependiendo de la

\footnotetext{
${ }^{6} \mathrm{Y}$ viceversa, los estados con menor crecimiento absoluto de su IPC estaban rodeados o eran vecinos de estados en una misma situación.

${ }^{7}$ Varios estados del sudeste y del norte, especialmente los fronterizos, mostraban poseer en 1990 más desviación estándar por encima de la media del IPC nacional.
} 
definición de vecinos, se puede obtener evidencia de procesos de cambio diferentes en el tiempo. Los cambios en el IPC no están concentrados espacialmente en el caso de estricta vecindad por contigüidad, pero para el caso de la vecindad por primer orden ajustado sí lo están, confirmando así que el crecimiento de un estado ha dependido espacialmente del crecimiento en: (a) estados contiguos, y (b) aquellos a una distancia menor al promedio.

\subsection{Sobre las variables explicativas de las desigualdades regionales en el largo plazo}

Este trabajo finalmente incluye el método de correlación RhoSpearman con el objetivo de probar asociaciones entre variables ordinales y lograr comparaciones en los rankings de IPC regionales. Por lo mismo, desde una perspectiva histórica a largo plazo, esta técnica puede ayudar a encontrar parcialmente las razones de las tendencias a la disparidad económica regional. Esta técnica es particularmente útil cuando la información económica no es abundante, y en este caso especialmente valiosa, ya que otorga a este estudio el beneficio de analizar casi un siglo de dinámica económica regional.

La fórmula de Correlación Rho de Spearman es la siguiente:

$$
o=1-\frac{6 \Sigma d^{2}}{n\left(n^{2}-1\right)}
$$

donde:

- $\quad d$ es la diferencia o resta entre los rankings de las unidades geográficas (estados) para cada año

- $n$ es el número de unidades geográficas (estados)

Las variables fueron obtenidas para cada estado $(\mathrm{N}=32)$, y se organizaron de manera ordinal; por ejemplo, empezando con el estado poseedor de la mayor tasa de alfabetización (1), hacia el estado con la menor (32) (véase el Cuadro 2).

El análisis de Lemoine (1973) muestra los coeficientes de correlación de las variables socioeconómicas de 1895 a 1960 con el IPC de 1960. En este estudio se ha continuado históricamente el análisis calculando los coeficientes de correlación Spearman para el periodo 1970 a 1990 con el IPC de 1990. De manera inicial se aprecia que el grado de asociación entre el IPC de 1980 con el IPC de 1990 es muy alto $(\mathrm{r}=.870, \mathrm{p}<.01)$. 
Los resultados de nuestro análisis son satisfactorios desde una perspectiva comparativa. Todas las variables coinciden con el trabajo previo de Lemoine en cuanto a su significancia estadística y en la dirección de la asociación. La única diferencia entre los coeficientes decenales son en su magnitud, lo cual hace a las variables consideradas en el estudio valiosas para explicar las causas de las desigualdades regionales.

Podemos observar que los niveles de urbanización del periodo 1970-1990 están fuertemente asociados con los niveles de IPC, pero menos que en el periodo previo de 1960 (véase el cuadro 8). La magnitud de los coeficientes de correlación se mantuvieron básicamente iguales entre 1980 y 1990, y muy similares para 1970. Es interesante observar que el IPC de 1960 no estaba significativamente asociado con el nivel de urbanización en 1900, en 1930 lo estuvo con una magnitud muy baja, y en 1960 lo estuvo en su mayor magnitud.

La inclusión de porcentaje de la fuerza de trabajo empleada en el sector Transporte es teóricamente reveladora. Con base en el estudio de Chinitz (1960), Lemoine sostuvo en su estudio que cuanto mayor fuera la importancia de este sector económico como proveedor de empleo, esto conduciría a mayores niveles de ingreso. Esta predicción teórica ha sido comentada por Katz (2000) en su análisis sobre la industria manufacturera mexicana y el TLCAN. La importancia de este sector en la economía es considerable y ha ido en aumento desde 1970 (de 4.9\% del PIB en 1970 a 7.9\% en 1995). Esta variable es estadísticamente significativa y fuertemente asociada a los niveles estatales de IPC para ambos años, 1960 y 1990. Su capacidad explicativa, sin embargo, parece haber disminuido entre 1980 y 1990.

La proporción de fuerza de trabajo empleada en agricultura parece haber tenido siempre una correlación negativa con el ranking estatal de IPC. También es observable una reducción en su importancia explicativa cuando se comparan los coeficientes de correlación del periodo 1940-1960 y el IPC de 1960 con los coeficientes del periodo 1980-1990 y el IPC de 1990. Aparentemente, la relación entre las dos variables no era tan fuerte en 1990 como lo fue en 1960. Aun así, su asociación negativa con el IPC es estadísticamente significativa y substancial.

Los niveles de alfabetismo siguen siendo importantes para explicar la variación regional en los niveles de IPC. Esto era de esperarse por dos razones principales. La primera es que esta variable tiene un peso teórico fundamental en historia económica. La se- 


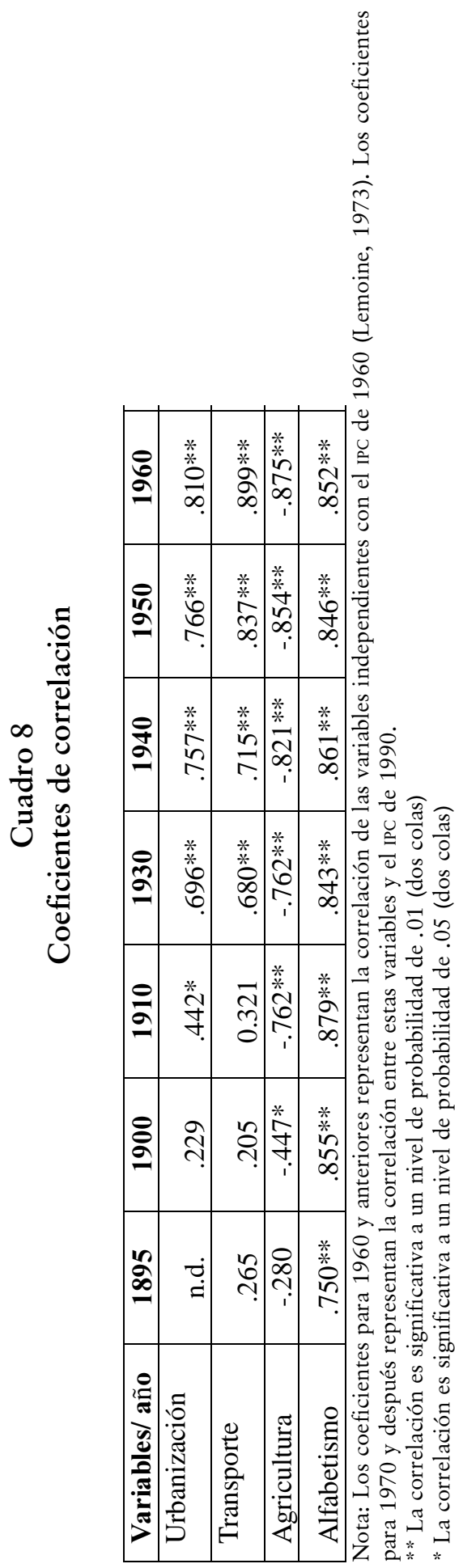


gunda es que esta variable está a su vez correlacionada con las previas variables independientes, por lo que su inclusión en un análisis de regresión puede ser erróneo metodológicamente hablando. Y como es el caso con las demás variables, la magnitud de los coeficientes de correlación muestran un decremento en los años ochenta. De hecho, el nivel de alfabetismo en una entidad está en 1990 menos asociado a su IPC inclusive que en 1895 .

En síntesis, en el periodo 1895-1960, la importancia de variables, como el transporte, la agricultura y el alfabetismo fueron aumentando en su capacidad para explicar el IPC de 1960. Sin embargo, comparativamente hablando, en el periodo 1980-1990 las mismas variables mostraron un decremento en su fuerza asociativa con el ranking estatal del IPC de 1990, aunque siguieron estando fuertemente asociadas. El nivel de urbanización como variable explicativa ha mantenido su importancia casi igual y por casi cien años.

\section{Discusión y conclusiones}

Para recapitular, Sala-i-Martín (1996), junto con otros, ha concluido que las economías desarrolladas tienden a converger en sus niveles de ingreso. Parte de su trabajo está fundamentado teóricamente, entre otros, en el análisis de Borts y Stein (1964) sobre la experiencia de los Estados Unidos. Quah (1996b) sostiene lo contrario: que las economías desarrolladas tienden a divergir. Por su parte, Myrdal (1957) predijo que el crecimiento económico no es equilibrado, sino que las regiones con ventajas iniciales tienden a distanciarse económicamente de las regiones más pobres, ${ }^{8}$ aunque también hay beneficios (spread-effects) para aquellas regiones vecinas. Este autor concluía que el papel del gobierno es fundamental para la compensación de esta tendencia a la divergencia.

Por razones teóricas e intereses metodológicos, utilizamos en este estudio tres técnicas diferentes para probar las hipótesis de convergencia regional de Sala-i-Martín (1996), y también para observar la capacidad explicativa de la teoría del crecimiento desbalanceado de Myrdal (1957). Las técnicas fueron: (1) regresión de mínimos cuadrados, (2) autocorrelación espacial, y (3) correlación de Spearman.

En primer lugar, los resultados sobre las pruebas de hipótesis de convergencia regional nos sugiere rechazar, primero, la hipó-

\footnotetext{
${ }^{8} \mathrm{Y}$ que esto ocurre con mayor efecto en las naciones menos desarrolladas
} 
tesis de convergencia sigma, en la que las diferencias en el ingreso tienden a descender en el tiempo. Concretamente, la evidencia muestra una tendencia a la divergencia regional durante el periodo 1970-1990. Para ser metodológicamente coherentes, se siguió de manera estricta la metodología de Messmacher (2000), y el análisis de regresión mostró una relación positiva muy pequeña entre la tasa de cambio en los niveles de IPC entre 1970 y 1990 con el IPC de 1970. Es decir, en este caso tampoco podemos aceptar la hipótesis de una convergencia de tipo beta, en donde las economías de los estados pobres tienden a crecer más rápido que las economías de los estados ricos. Por lo tanto, con base en la evidencia y la metodología utilizada, se concluye en este trabajo que los estados mexicanos en su conjunto -es decir, no espacializando el análisis- han tendido a distanciarse en sus niveles de IPC durante el periodo analizado. Los estudios de Lemoine (1973) y Hernández (1984) muestran que esto ya venía sucediendo desde comienzos del siglo xx.

En segundo lugar, el análisis espacial del IPC nos muestra resultados diferentes según el tipo de datos que utilicemos. Los coeficientes de autocorrelación espacial sugieren una tendencia, en este caso, a la disminución en las disparidades regionales durante 1970 a 1990. ${ }^{9}$ También se mostró evidencia de que la tasa de cambio en el IPC de los estados estuvo asociado espacialmente de forma positiva. Es decir, el crecimiento fue concentrado o espacialmente dependiente en algunas regiones; ocurrió en aquellos estados que eran cercanos a otros estados también en crecimiento. Esto concuerda con lo encontrado en Europa por Quah (1996b) al respecto de los clubes de convergencia, en donde el mayor desarrollo ocurre geográficamente concentrado. Estos hallazgos comprueban que México es claramente un país de regiones económicas unidas políticamente, que muestra una geografía del crecimiento; hay clubes de convergencia, por ejemplo Campeche-Quintana Roo-Tabasco o Sonora-Coahuila-Chihuahua. Hay estados que si bien no retroceden en sus niveles de IPC, no están conectados ni funcional ni geográficamente con el desarrollo que se presenta en otros estados.

Finalmente, Lemoine (1973) con base en la tesis de Myrdal (1957) argumentaba que las desigualdades regionales se debían a velocidades diferentes entre los estados mexicanos en sus proce-

${ }^{9}$ Esto se demostró con dos tipos de vecindad geográfica: de primer orden y de primer orden ajustado (véase el cuadro 7 y su explicación en el mismo apartado). 
sos de urbanización e industrialización. A este respecto, los resultados de nuestro estudio de largo plazo muestran que la tendencia y la fuerza de asociación entre las variables no es la misma para el periodo 1895-1960 que para el periodo 1970-1990. La comparación de estos dos espacios temporales indica que la importancia de las variables de Lemoine decrecieron en su capacidad explicativa. La prueba estadística fue la misma. La cuestión radica en explicar qué tan diferentes fueron las décadas de los setenta y ochenta con el periodo previo.

A este respecto, primero hay que considerar que durante los ochenta, en México ocurrió una de las peores crisis económicas experimentadas. Además de esto, una importante transformación económica se llevó a cabo hacia una terciarización de la economía (54.5 en 1970 a 60\% del PIB en 1990) lo cual tiene implicaciones muy importantes en términos de empleo e ingreso. Esta transformación vino en detrimento del sector agrícola (11.2 al 7.6\% del PIB para el mismo periodo) y el sector manufacturero, que también perdió algo de su previa importancia (22.5\% en 1970 a $18.4 \%$ en 1990).

Como Garza y Rivera (1994) y Arroyo (2000) explican, en correspondencia con la crisis económica de los ochenta hubo también una redistribución de las actividades económicas entre las regiones Mexicanas. El norte y la península de Yucatán sufrieron menos los efectos de la crisis, y de hecho aumentaron su IPC a través del crecimiento eficiente de la industria maquiladora en la frontera, y del turismo y la extracción petrolera en la península. En cambio, el sur y el centro de México, más rural y tradicionalmente industrial, respectivamente, vieron dañar su economía en la recesión de la década y su IPC promedio irse abajo en el ámbito nacional.

Es interesante observar, también, que entre 1980 y 1990 la velocidad en las tasas de urbanización mostró una desaceleración (de 56.2\% a $60.8 \%$ ), mientras que en décadas pasadas estas tasas cambiarían mas rápidamente (Garza y Rivera, 1994). También debe considerarse que de acuerdo con los hallazgos de Lemoine, durante esta última década el nivel de urbanización mostró ser una razón de desigualdad regional en el IPC; en promedio, avances en los niveles de IPC se presentaron en los estados más urbanizados.

De manera concluyente, la apreciación que se desprende de este estudio es principalmente metodológica, y es que se define claramente como indispensable la inclusión de las técnicas de aná- 
lisis espacial en el estudio de las desigualdades regionales. Se llegó a esta conclusión a través de la prueba de diversas hipótesis con técnicas diferentes. Así pues, la utilización de los coeficientes de autocorrelación de Morán fue acertada, en el sentido de que puso de relieve la importancia de la dimensión geográfica del proceso económico. Si bien a través del análisis de regresión encontramos una tendencia a la dispersión en el ingreso, con el análisis espacial nos dimos cuenta de que esta dispersión no era registrada con la utilización de dos matrices diferentes de estados vecinos. Al contrario, se detectó una nivelación espacial en los niveles de IPC. El caso de la inclusión del estado de Campeche en el sudeste, con su crecimiento notable durante el periodo estudiado, fue un factor fundamental en el resultado. Sin embargo, debe apuntarse que el registro de una tasa de cambio concentrada espacialmente nos indica que los crecimientos relativos se encontraban concentrados geográficamente en clusters, aunque fue en estados geográficamente distantes donde se registraron los cambios más notables (Campeche, Colima, Zacatecas), lo cual afecta el cómputo del coeficiente.

A este respecto metodológico, es recomendable en próximos estudios dirigirse a niveles más detallados, tales como ciudades, e incluir variables explicativas directamente en los análisis de regresión. De la continuación del análisis de Lemoine (1973) se pudo concluir que el periodo asociado con la crisis de los ochenta puede ser fácilmente identificado como diferente respecto del pasado. Por lo tanto, teorías geográficas y explicaciones diferentes ad hoc para cada periodo histórico deben ser puestas a prueba.

\section{Bibliografía}

Anselin, L. (1988), Spatial Econometrics: Models and Methods, Kluwer Academic Publishers, Dorddrecht.

Arroyo, Francisco (2001), "Dinámica del PIB de las entidades federativas de México, 1980-1999”, Comercio Exterior, vol. 51, núm. 7, México.

Borts, G. y Jerome Stein (1964), Economic Growth in a Free Market, Columbia University Press, New York. 
Carlino, G. \& Mills, L. (1996), “Testing neoclassical convergence in regional incomes and earnings", Regional Science And Urban Economics, Vol. 26 (6), pp. 565-590.

Chinitz, B. (1960), "The effect of transportation forms on regional economic growth", Traffic Quarterly, Vol. 14, pp. 129142.

Coelho, P. \& M. Ghali (1971), “The end of the North-South wage differential”, American Economic Review, 61, pp. 932-937.

Fujita, M. \& T. Tabuchi (1997), "Regional growth in postwar Japan”, Regional Science And Urban Economics, Vol. 27 (6), pp. 643-670.

Garza, G. \& S. Rivera (1994), Dinámica Macroeconómica de las Ciudades en México, INEGI, ColmeX e IIS-UnAm, México.

Hall, P. \& D. Hay (1980), Growth Centres in the European Urban System, Heinemann, London.

Hernández, E. (1984), "La Desigualdad Regional en México (1900-1980)", en R. Cordera y C. Tello (Coords.), La Desigualdad en México, 2a. edición, Siglo XXI Editores, México, pp. 155-192.

Hoover, E. \& F. Giarratani (1984), An Introduction to Regional Economics, 3rd edition, Alfred A. Knopf, New York.

Katz, I. (2000), "El impacto regional del Tratado de Libre Comercio de América del Norte: Un análisis de la industria manufacturera", en Beatriz Leycegui y Rafael Fernández (eds.), TLCAN ¿Socios Naturales? Cinco Años del Tratado de Libre Comercio de América del Norte, ITAM, México.

Kollman, R. (1995), "The correlation of productivity growth across regions and industries in the United States", Economics Letters, Vol. 47 (3-4), pp. 437-443.

Leach, J. (1996), “Training, migration, and regional income disparities", Journal of Public Economics, Vol. 61 (3), pp. 429-443. 
Lemoine, J. (1973), "Causación acumulativa y crecimiento interregional de México”, en Leopoldo Solís (Sel.), La Economía Mexicana: Política y Desarrollo, Fondo de Cultura Económica, México, pp. 541-573.

Magrini, S. (1999), “The evolution of income disparities among the regions of the European Union”, Regional Science And Urban Economics, Vol. 29 (2), pp. 257-281.

Messmacher, Miguel (2000), "Desigualdad regional en México. El efecto del TLCAN y otras reformas estructurales", Documento de Investigación núm. 2000-4, Dirección General de Investigación Económica, Banco de México, disponible en: <http://www.banxico.org.mx/gPublicaciones/DocumentosInvestigacion/docinves/doc2000-4/doc20004.pdf $>$

Morán, P. (1950), "Notes on continuous stochastic phenomena”, Biometrika 37, pp. 17-23.

Myrdal, G. (1957), Economic Theory and Under-Developed Regions, Duckworth, London.

Paci, R \& F. Pigliaru (1997), "Structural change and convergence: an Italian regional perspective", Structural Change and Economic Dynamics, Vol. 8 (3), pp. 297-318.

Puga, D. (1999), “The rise and fall of regional inequalities”, European Economic Review 43(2), pp. 303-33.

Persson, J. (1997), "Convergence across the Swedish counties, 1911-1993", European Economic Review, Vol. 41 (9), pp. 1835-1852.

Quah, D. (1996a), "Regional convergence clusters across Europe", European Economic Review, Vol. 40 (3-5), pp. 951958.

- (1996b), "Empirics for economic growth and convergence”, European Economic Review, Vol. 40 (6), pp. 1353 1375 . 
Sala-i-Martín, X. (1996), "Regional cohesion: Evidence and theories of regional growth and convergence", European Economic Review, Vol. 40 (6), pp. 1325-1352.

Vilalta, C. (2003), "Measuring the Spatial Clustering and Diffusion of Multiparty Competition in Urban Mexico (19942000)", ponencia presentada en el International Research Workshop, mayo 28-31, Center for New Institutional Social Sciences, Washington University, St. Louis Mo, disponible en: < http://cniss.wustl.edu/internationalresearchworkshoppapers/vilalta.pdf $>$

Enviado: 20 de febrero de 2003. Reenviado: 24 de julio de 2003. Aceptado: 1 de agosto de 2003. 